\title{
Physico-chemical, functional and rheological characterization of biodegradable pellets and composite sheets
}

\author{
Kulsum Jan ${ }^{a}$, Shumaila Jan, CS Riar and DC Saxena \\ Department of Food Engineering and Technology \\ Sant Longowal Institute of Engineering \& Technology, Longowal - 148 106, Punjab, INDIA
}

\begin{abstract}
Deoiled rice bran, paddy husk, cashew nut shell liquid and glycerol were extruded into pellets and further pressed into composites. Processing and plasticizer type had significant effect on physico-chemical, functional, rheological and morphological properties of pellets and composites. Specific mechanical energy of the pellets containing cashew nut shell liquid as plasticizer was higher than those containing glycerol. The maximum hardness and bulk density were obtained for pellets prepared from cashew nut shell liquid. Water binding capacity and water solubility index of both pellets and composites were highest for samples containing glycerol as plasticizer. A significant change in functional properties during processing was observed among raw materials, pellets and the final product (composite sheets).
\end{abstract}

Key words: Biodegradation, Cashew nut shell liquid, glycerol, plasticizer, functional, rheological.

\section{Introduction}

Biodegradable polymers are substitutes to the synthetic polymers which have occupied dangerous proportions among the environmental pollutants. Wastes from agroindustries generated during the industrial processing of agricultural products [1] have issues with their disposals. These wastes and byproducts are rich in minerals, sugars and proteins and can be used as raw materials for other industrial processes and development of value added products [2]. In the past most of the edible scraps were utilized for the production of animal feed, compost and fertilizer; otherwise they were discarded in common dumps. In order to reduce the trash collection and disposal fees, different methods and policies for byproduct and waste handling and treatment have been introduced to recover, recycle and convert the byproducts and wastes into upgraded products [3]. Biocomposites from natural fibers and biodegradable polymers have grabbed great interests in the composite science [4].

Deoiled rice bran a major byproduct generated during rice milling and subsequent oil extraction is a valuable source of inexpensive protein, contains about 12$20 \%$ and is an underutilized agro industrial byproduct [5]. Besides deoiled rice bran, paddy husk is one of the major agricultural residues produced during rice processing containing cellulose in similar amounts with wood. Usually paddy husk has been a problem for rice farmers due to its resistance to decomposition in the ground, difficult digestion, inadequate final disposal (burning) and low nutritional value for animals. The lignin and hemicellulose contents of paddy husk are lower than wood, for this reason paddy husk can be processed at higher temperatures than wood. Cashew nut shell is another waste product of cashew nut industry it is of great industrial importance and has recently been used to produce biocomposites [6].

Technologies like extrusion have found its application in other sectors like packaging technology besides being used for development of food products. The thermal energy generated by viscous dissipation during extrusion, combined with shearing effect, quickly cooks the raw mixture so that the properties of materials are modified due to physico-chemical changes of the biopolymers [7]. The effect of extrusion on the properties of pellets, using rice husk as filler was analyzed by Simone [8]. Zein based pellets were also developed by Wu et al. [9]. Till date, research is scanty on properties of pellets developed from deoiled rice bran and potato peel, the agro industrial byproducts and wastes along with plasticizers such as cashew nut shell liquid and glycerol using extrusion technology. Hence, the main aim of our study is to develop and characterize the pellets from extrusion for the development of sheets. The sheets were also characterized in second part of the study.

\section{Materials and methods}

\subsection{Procurement of raw materials}

Paddy husk and deoiled rice bran used for the present study were donated by M/s. AP Solvex Ltd., Dhuri (Punjab, India). Analytical grade glycerol was used as plasticizer (M/s. Merck Specialities Pvt. Ltd., Mumbai,

\footnotetext{
${ }^{a}$ Corresponding author: kulsumnissar@gmail.com
} 
India). Cashew nut shell liquid was kindly donated by M/s. Allen Petrochemicals Pvt Lt., Meerut (India).

\subsection{Sample preparation}

Paddy husk was ground in a Laboratory grinding mill (M/s. Philips India Limited, Kolkata, India) and passed through 60 mesh screen to obtain fine powder. Similar process was followed for deoiled rice bran.

\subsection{Physico-chemical and functional properties of raw materials}

\subsubsection{Proximate composition of raw materials}

The raw materials were analysed for moisture, protein, fat, fibre and ash content according to AOAC [10].

\subsubsection{Specific mechanical energy (SME) of optimized pellets}

The specific mechanical energy (SME) of extrusion was calculated by following eqn.:

$\mathrm{SME}=\left(\omega / \omega_{\mathrm{r}}\right) \times(\tau / 100) \times\left(\mathrm{Z}_{\mathrm{r}} \mathrm{Q}\right)$

where $\omega$ is screw speed, $\omega_{\mathrm{r}}$ is the rated screw speed, $\tau$ is percent torque, $Z_{\mathrm{r}}$ is rated power, and $\mathrm{Q}$ is the feed rate.

\subsection{Development of pellets and sheets}

Deoiled rice bran (DOB), paddy husk powder (PDH), cashew nut shell liquid (CNSL) and glycerol (GL) were used in the blend preparation to prepare pellets. Different formulations used were (DOB, PDH and CNSL) and (DOB, PDH and GL). All the ingredients were weighed and mixed in a Hobart mixer (M/s. Continental Equipment India Pvt. Ltd, Delhi, India). Extrusion of the blends was performed using a co-rotating twin-screw extruder (M/s. Basic Technology Pvt. Ltd., Kolkata, India) under suitable conditions (Process filed for patent, No: 111/DEL/2015). The pellets prepared from extrusion were molded in hydraulic press to form sheets which were then stored for further analysis.

\subsection{Characterization of pellets and composite sheets}

\subsubsection{Physical and mechanical properties}

\subsubsection{Color of pellets and sheets}

Color measurements of all the pellets were carried out in triplicate as per standard protocol using a hand-held Chroma Meter (CR-400, Konica Minolta, UK) and L*, $\mathrm{a}^{*}$, and $\mathrm{b}^{*}$ values were recorded. The values of $\Delta \mathrm{E}$ were calculated as:

$$
\Delta E=\sqrt{\left(L^{*}-L\right)^{2}+\left(a^{*}-a\right)^{2}+\left(b^{*}-b\right)^{2}}
$$

where $L^{*}$ is the standard value and $L$ is the obtained value. Similarly, $a^{*}$ and $b^{*}$ are the standard values and $a, b$ are the obtained values.

\subsubsection{Bulk density of pellets and sheets}

The density was measured as per procedures described by Jan et al. [7].

\subsubsection{Frictional properties of pellets}

The angle of repose is very important in characterization of the flow properties of pellets. The static coefficient of friction of the pellet against the various surfaces is used to determine the angle at which the pellets must be positioned in order to achieve consistent flow of pellets. A wooden table provided with an adjustable tilting plate on its top, facing to the test surface, was used for the purpose. The sample was kept on adjustable plate placed on top of the table and the inclination of the test surface was increased gradually so that the pellets started to slide down until about $75 \%$ of the pellets slided down. The static coefficient of friction $(\mu)$ was calculated from the following equation:

$$
\mu=\tan \alpha
$$

The angle of repose is derived from the following equation

$$
\theta=\tan ^{-1}\left\{\frac{2 H}{D}\right\}
$$

Where, $\mathrm{H}$ and $\mathrm{D}$ are the height and diameter of the heap, respectively.

\subsubsection{Hardness of pellets and sheets}

A crushing method using a TA - XT2i Texture Analyzer (M/s. Stable Micro Systems Ltd., Godalming, UK) was used to determine the hardness of pellets. Five pellets from each sample were mounted on the platform of the analyzer with a probe $\mathrm{P} / 75-75 \mathrm{~mm}$ diameter at a crosshead speed of $2 \mathrm{~mm} / \mathrm{sec}$ with a target mode of $2 \mathrm{~mm}$ distance. The highest first peak value was recorded as hardness.

\subsubsection{Pellet durability index}

Durability of pellets was determined using the two compartments pellet-durability tester (M/s. Continental Agra Equipment Inc, India) [7].

\subsubsection{Flexural properties of pellets and sheets}

The flexural test was conducted at different storage conditions (relative humidity-RH and temperature) mentioned below [9]:

1. Dry state: under ambient conditions; 2. $20 \%$ RH state: equilibrated at $25^{\circ} \mathrm{C}$ and $20 \% \mathrm{RH}$ for 7 days; 3. $75 \% R H$ state: equilibrated at $75 \% \mathrm{RH}$ for 7 days; $4.95 \% \mathrm{RH}$ state: equilibrated at $95 \% \mathrm{RH}$ for 7 days.

Maximum flexural stress $\left(\sigma_{\mathrm{m}}\right)$ was calculated as following equation:

$$
\sigma_{m}=\frac{3 \mathrm{PL}}{2 B d^{2}}
$$

where $\mathrm{P}$ is the maximum stress, ' $\mathrm{L}$ ' is the support span, ' $\mathrm{B}$ ' is the width and ' $\mathrm{d}$ ' is the thickness of the pellet.

\subsection{Functional properties of pellets and sheets}

\subsubsection{Water binding capacity (WBC)}

A suspension of $5 \mathrm{~g}$ ground sample (dry weight) in $75 \mathrm{ml}$ distilled water was agitated for $30 \mathrm{~min}$ at $25^{\circ} \mathrm{C}$ and centrifuged $(3000 \times \mathrm{g})$ for $10 \mathrm{~min}$. The supernatant was discarded, drained for $5 \mathrm{~min}$ and the gain in weight was expressed as percentage of water binding capacity.

\subsubsection{Water solubility index (WSI)}


WSI of raw materials were measured using a technique described by Anderson [11].

\subsubsection{Water resistance and water uptake}

The water resistance of injection-molded pots was measured by using the method as described by $\mathrm{Wu}$ et al. [9] with slight modifications. The experiment was carried out in triplicates. The percentage change in weight was plotted against time.

The water uptake of injection-molded pots was measured by using the method described by Schettini et al. [12] with slight modifications. The experiment was carried out in triplicates. The water up-take percentage was calculated using the equation:

$$
\text { Water uptake }(\%)=\frac{W d-W t}{W d} \times 100
$$

Where, $\mathrm{Wd}$ and $\mathrm{Wt}$, respectively are the weights of the samples in the dry state and in the swollen states at different times.

\subsection{Statistical analysis}

The data reported represent average of triplicate observations \pm standard deviation. Statistical analysis was performed using Statistica-log software package version 7 (M/s. StatSoft Inc., OK, USA). The significant differences were obtained by a one-way analysis of variance (ANOVA) followed by Duncan's multiple range test (DMRT) $(\mathrm{P}<0.05)$.

\section{Results and discussion}

\subsection{Proximate analysis}

The proximate analysis (14\% moisture basis) of raw materials revealed that DOB contained ash $7.94 \%$, protein $13.78 \%$, fat $0.63 \%$, crude fibre $8.26 \%$ and carbohydrates $69.37 \%$. PDH contained $12.25 \%$ ash, $0 \%$ protein, $0 \%$ fat, crude fibre $29.81 \%$ and carbohydrates $63.52 \%$. It was observed that DOB has colour values $(\Delta \mathrm{E})$ as 33.588 and $\mathrm{PDH}$ has 31.327 which are quite significantly different. The water binding capacity (WBC) of DOB and PDH was found to be 3.52 and $3.94 \mathrm{~g} / \mathrm{g}$, respectively. Water solubility index (WSI) of the DOB and PDH was 10 and $16 \%$, respectively. WBC of raw materials provides an insight of the ability of protein structure to associate with hydrophilic substance (water), the bound water which can further function as plasticizer during different processing operations.

Table 1. Effect of plasticizers on physical, functional and mechanical properties of pellets

\begin{tabular}{|c|c|c|c|c|}
\hline \multirow{2}{*}{$\begin{array}{l}\text { Pellets } \\
\text { properties }\end{array}$} & \multicolumn{2}{|c|}{ CNSL } & \multicolumn{2}{|c|}{ Glycerol } \\
\hline & Pellets & Sheets & Pellets & Sheets \\
\hline SME (kJ/kg) & $69.817^{b}$ & & $61.922^{\mathrm{a}}$ & \\
\hline \multirow{2}{*}{$\begin{array}{l}\text { ER } \\
\text { PDI (\%) } \\
\text { AOR }\left(^{\circ}\right)\end{array}$} & $1.10^{\mathrm{a}}$ & & $1.19^{\mathrm{b}}$ & \\
\hline & $99.040^{\mathrm{b}}$ & & $98.750^{\mathrm{a}}$ & \\
\hline $\operatorname{AOR}\left({ }^{\circ}\right)$ & $50.45^{\mathrm{a}}$ & & $51.34^{\mathrm{b}}$ & \\
\hline & $0.422^{\mathrm{a}}$ & & $0.482^{\mathrm{b}}$ & \\
\hline WD & $0.709^{\mathrm{a}}$ & & $0.752^{\mathrm{b}}$ & \\
\hline Hardness $(\mathrm{N})$ & $453.38^{\mathrm{b}}$ & $480.25^{\mathrm{b}}$ & $448.58^{\mathrm{a}}$ & $477.85^{\mathrm{a}}$ \\
\hline
\end{tabular}

\begin{tabular}{lllll}
\hline Color $(\Delta \mathrm{E})$ & $52.416^{\mathrm{b}}$ & $54.711^{\mathrm{b}}$ & $49.316^{\mathrm{a}}$ & $48.54^{\mathrm{a}}$ \\
WBC $(\mathrm{g} / \mathrm{g})$ & $5.34^{\mathrm{a}}$ & $4.07^{\mathrm{a}}$ & $6.14^{\mathrm{b}}$ & $0.685^{\mathrm{b}}$ \\
WSI $(\%)$ & $17.54^{\mathrm{a}}$ & $11.82^{\mathrm{a}}$ & $20.34^{\mathrm{b}}$ & $14.72^{\mathrm{b}}$ \\
Density $(\mathrm{g} / \mathrm{ml})$ & $0.735^{\mathrm{b}}$ & $1.21^{\mathrm{b}}$ & $0.685^{\mathrm{a}}$ & $1.13^{\mathrm{a}}$ \\
\hline
\end{tabular}

Means with small letters in a column differ significantly $(\mathrm{p}<0.05)$

\subsection{Specific mechanical energy (SME)}

As shown in Table 1, a significant difference in SME was observed between the pellets developed from both the blends. Highest value of SME was observed for sample containing CNSL than the sample containing glycerol. This may be due to the more binding effect of the plasticizer (CNSL) in the first stage with an exposure of hydrophilic sites, reinforcing the cross linkage. Thereafter, the network structure might have been formed with covalent crosslinks and reduced molecular size due to increased mechanical shear.

\subsection{Color of pellets and sheets}

Color is a physical property that may change during the extrusion cooking process [13]. A significant change was observed before and after extrusion as shown in Table 1 . The change may be attributed to the extruder temperature and screw speed resulting in varying the structure and stir up browning reactions [14]. According to Rosentrater [13], Maillard reactions and protein denaturing play main roles in colour alterations during the cooking process resulting from high temperatures. It was also observed that the color $(\Delta \mathrm{E})$ value of samples containing CNSL as plasticizer were greater than samples containing glycerol as plasticizer which is due to the inherent dark color of the cashew nut shell liquid [7], resulting in maximum absorption of the light. In case of sheets, a significant increase in colour $(\Delta \mathrm{E})$ was observed during pressing (hydraulic press) which may be due to higher temperature during processing as compared to that of pellets during extrusion.

\subsection{Density and hardness of pellets and sheets}

The density of pellets with CNSL and glycerol was found to be 0.738 and $0.685 \mathrm{~g} / \mathrm{ml}$, respectively (Table 1). However, the density of sheets containing CNSL and glycerol was 1.216 and $1.136 \mathrm{~g} / \mathrm{ml}$, respectively. It is evident from the results that the density of both the pellets and sheets were higher for samples with CNSL. It may be due to the reduced porosity and availability of binding sites in CNSL to bind with the raw material.

The textural property (hardness) of pellets was determined by measuring the force required to break the pellet. The higher value of peak force required to breakdown the sample means higher the hardness of the sample to get fractured. It was observed that the hardness of the samples (CNSL) were greater than samples (glycerol). The increased hardness may be attributed to the greater binding effect of CNSL as compared to glycerol. Moreover, the increased hardness measured by texture analyser is due to more resistance to compression [7]. Plasticizers are important to maintain dimensional stability, hardness and flexibility of protein-based 
polymer due to its high boiling temperature [9]. The hardness was found to be directly correlated with the density of the pellets.

\subsection{Frictional properties of pellets}

A significant difference was observed between angle of repose (AOR) of both the samples (Table 1). The angle of repose for sample with CNSL was lesser than the samples with GL which may be due to the free flowing nature of pellets with CNSL which encourages the down slope movement of pellets making them buoyant and accordingly reducing angle of repose. The reduced angle of repose may be due to the smooth surface of samples (CNSL) which may be attributed to the improved binding effect of CNSL than glycerol. The parameter is important in design of hoppers to maintain continuous flow of the pellets, which must be larger than the angle of repose of the pellets [15].

The static coefficient of friction for galvanized iron (GI) surface was lesser for both the cases as compared to wooden surface (Table 1). However, COF for CNSL pellets was lower as compared to GL pellets. The reduction in coefficient of friction may be explained similarly as in the case of angle of repose. Moreover, the higher density of sample (CNSL) can also take part in reduction of friction during flow. Coefficient of friction is used to determine the angle at which the chutes must be positioned to achieve a consistent flow of the pellets [16]. The results indicate that galvanized iron can serve as a better medium for conveying pellets during processing.

\subsection{Pellet durability index}

PDI is another important physical property of the pellets. It indicates the mechanical strength of the extruded products (pellets). The higher the PDI, the more stable the extrudates during storage and handling processes. As shown in Table 1, The PDI for both the cases exceeded the standard durability index $(97.5 \%)$ set for handling and transportation of pellets by British Standards Institution [17]. The increased hardness and durability can be attributed to the strong binding effect of the plasticizer causing strong interactions between particles that are similar to those of solid bridges [18].

\subsection{Flexural properties of pellets and sheets}

The effect of plasticizer level on the flexural strength in all the storage conditions is shown in Fig 1. In the dry state, for both the cases (GL, CNSL) sheets showed a maximum strength due to the low moisture content as compared to other conditions. At the $20 \% \mathrm{RH}$ state, the water content increased more on an average than that in the dry state, and flexural strength decreased from an average of $128.670 \mathrm{MPa}$ for the dry state to an average of 47.367 MPa for GL-sheets. However, in case of CNSLsheets a decrease from $134.250 \mathrm{MPa}$ to $54.874 \mathrm{MPa}$ was observed. It is obvious from results that the strength of the CNSL-sheets was greater than GL-sheets which may be due to the increases hardness and reduced porosity of CNSL sheets. Increased porosity will result in imbibitions of water.

\subsection{Water binding capacity and water solubility index}

Water binding capacity of pellets with CNSL was less than that of samples containing glycerol which may due to the plasticizing effect of polyols (glycerol), in particular attributed to their ability to locate between the chains of proteins, bind water, and disrupt intermolecular polymer interactions [19]. Moreover, it may be also due to the hydrophilic and hygroscopic nature of glycerol and its ability to form the large hydrodynamic plasticizerwater complex [7].

Similar behaviour of glycerol has been observed in films developed using glycerol as plasticizer due to the hygroscopic character of plasticizers [20-21]. A significant change in water binding capacity was observed among the raw materials and the pellets formed. This change can be attributed to rupture of the associations by heat and shear during extrusion. In case of the sheets, a similar trend of WBC was observed for both the samples (Table 1). Pellets and sheets also showed a significant difference between them (Table 1).

A significant change in water solubility index among the raw materials, pellet and sheets was observed which can be explained by structural modifications of the feed compositions during extrusion [13, 21]. WSI of pellets (CNSL) was lower than that of samples with

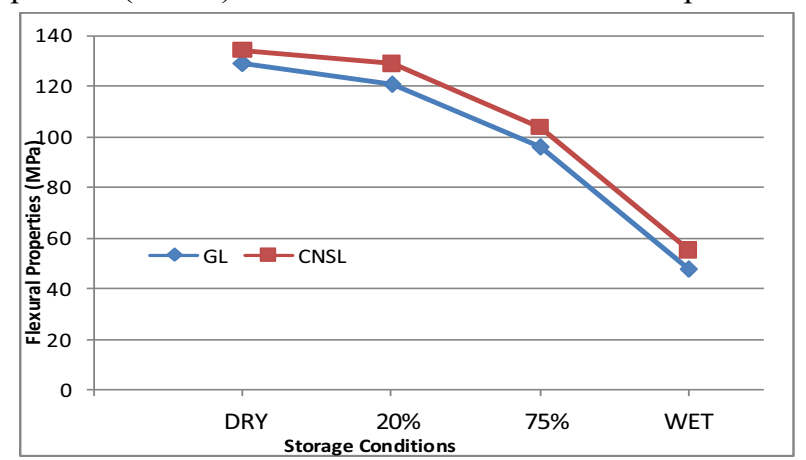

Figure 1. Effect of storage condition on flexural properties of sheets

glycerol (Table 1) which may be due to immiscible nature of CNSL with water. A similar trend of WSI was observed for sheets as in case of pellets. Hence, it can be predicted that processing plays an important role in affecting the functional properties of the pellets and pots.

\subsection{Water resistance and water uptake}

Water leaked slowly out of the pots during the whole test of water resistance of pots as shown in Fig. 2. The weight loss observed in CNSL-sheet was significantly lower than GL-sheets. In case of CNSL-sheets, a weight loss of $1.32 \%$ was observed for first $5 \mathrm{hrs}$ which rose to $3.24 \%$ after $10 \mathrm{hrs}$. Initially, a gradual weight loss was observed which rose to $27.20 \%$ after $30 \mathrm{hrs}$. Similarly, in case of GL-sheets a weight loss of $2.06 \%$ was observed for first 5 hrs which rose to $28.0 \%$ after $30 \mathrm{hrs}$. Increased weight loss of GL-sheets may be due to the increased porosity and more free spaces available for water to penetrate into the walls of the GL-sheets. 
GL-sheets experienced a faster water uptake (16.93\%) mainly during the first 15 minutes of water dipping, absorbing most of the water in early stages then followed by a gradual increase rising upto $33.42 \%$ of water uptake after $60 \mathrm{~min}$. However, in contrast to GLsheets, CNSL-sheets absorbed $12.26 \%$ in first $15 \mathrm{~min}$ which rose upto $30.09 \%$ after $60 \mathrm{~min}$.

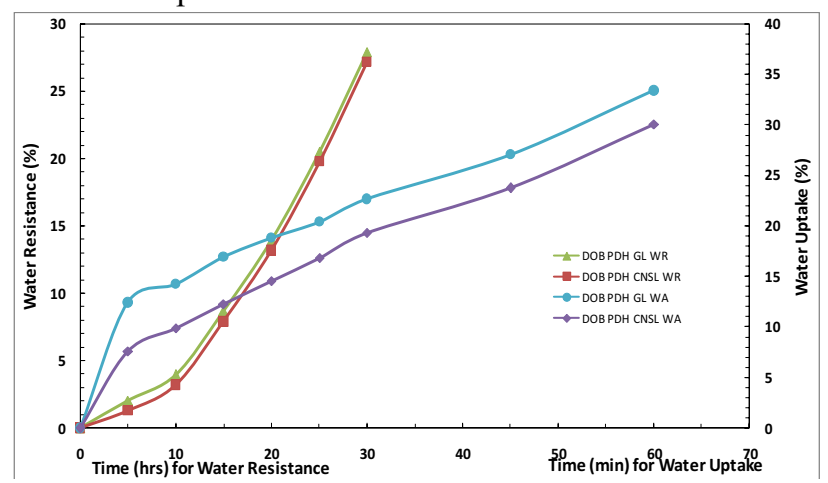

Figure 2. Effect of plasticizers on water resistance and water uptake of sheets

\section{Structural data analysis}

The factor loading obtained for the first two components (PCs) and the percentage of variance along with cumulative variance in case of pellets and sheets is shown in Table 2. In case of sheets, the first two principal components accounted for $99.583 \%$ of the variance. The first and second principal components (F1 and F2) explained 79.332 and $22.251 \%$ of the variance, respectively. It was observed that F1 was highly positively correlated with water solubility index and negatively correlated with water binding capacity and density. Second principal component F2 was highly negatively correlated with hardness. It was observed from Fig. 3 that CNSL-sheets were positioned on left side of principal component 1 (F1) which were linked to density and water binding capacity. However, GL-sheets were also positioned on left side of principal component 1 (F1) which were linked to hardness and color.

Table 2. Principal component analysis: loading of first two components for properties of pellets and sheets

\begin{tabular}{lll}
\hline Sheets & F1 & F2 \\
\hline Eigen value & 3.967 & 1.013 \\
Variability (\%) & 79.332 & 20.251 \\
Cumulative \% & 79.332 & 99.583 \\
\hline Factor loadings & $\mathbf{F 1}$ & $\mathbf{F 2}$ \\
\hline WBC & $\mathbf{- 0 . 9 9 5}$ & 0.033 \\
WSI & $\mathbf{0 . 9 9 5}$ & -0.017 \\
Hardness & -0.408 & $\mathbf{- 0 . 9 1 3}$ \\
Density & -0.908 & 0.418 \\
Color & $\mathbf{- 0 . 9 9 7}$ & -0.057 \\
\hline Pellets & $\mathbf{F 1}$ & $\mathbf{F 2}$ \\
\hline Eigen value & 6.500 & 2.377 \\
Variability (\%) & 72.198 & 26.413 \\
Cumulative \% & 72.198 & 98.610 \\
\hline Factor loadings & $\mathbf{F 1}$ & $\mathbf{F 2}$ \\
\hline Hardness & $\mathbf{- 0 . 9 3 3}$ & 0.360 \\
WBC & 1.000 & -0.001 \\
Color & $\mathbf{- 0 . 9 2 6}$ & 0.378 \\
Pellet Durability Index & -0.844 & -0.451
\end{tabular}

\begin{tabular}{lll} 
Water Solubility Index & $\mathbf{0 . 8 4 4}$ & -0.520 \\
Coefficient of Friction (GI) & $\mathbf{0 . 8 1 2}$ & 0.579 \\
Coefficient of Friction (WD) & $\mathbf{0 . 9 4 4}$ & 0.325 \\
Angle of Repose & 0.684 & -0.727 \\
Density & 0.573 & $\mathbf{0 . 8 1 3}$ \\
\hline
\end{tabular}

In case of pellets sheets the first two principal components accounted for $98.610 \%$ of the variance. The first and second principal components (F1 and F2) explained 72.198 and $26.413 \%$ of the variance, respectively. It was observed that F1 was highly positively correlated with water solubility index, coefficient of friction (both wood and galvanized iron) and highly negatively correlated with color and hardness. It was observed from Fig.4 that CNSL-sheets were positioned on right side of principal component 1 (F1) which were linked to density, coefficient of friction (both wood and galvanized iron), water binding capacity, angle of repose and water solubility index. However, GL-sheets were positioned on left side of principal component 1 (F1) which were linked to pellet durability index, color and hardness. Hence, on the basis of these parameters GLsheets can be differentiated from CNSL-sheets.

\section{Conclusion}

It was observed from the results that sheets prepared from CNSL were having more water resistance and hardness than those prepared from glycerol. A significant change was observed between the raw materials pellets and sheets. On the basis of results obtained for sheets it was concluded that pellets with the same formulation can be used for the preparation of various molded products at high temperatures.

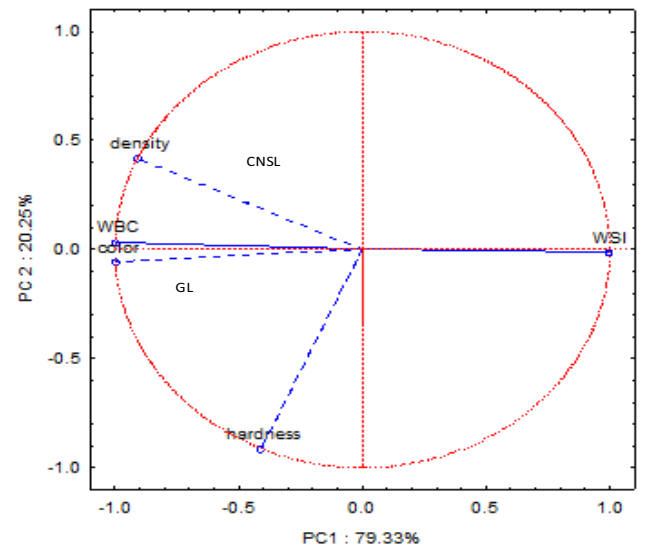

Figure 3. Principle component score plot for sheets 


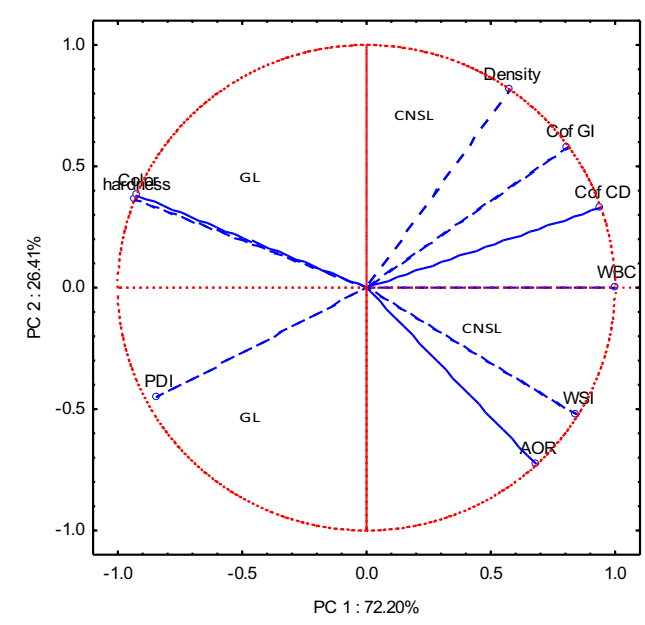

Figure 4. Principle component score plot for pellets

\section{Acknowledgments}

Financial support from Council of Scientific and Industrial Research (CSIR), New Delhi, Govt. of India is gratefully acknowledged.

\section{References}

1. Singh, S., 2014. Production and characterization of industrially important amylase enzyme by Aspergillus niger using different combination of starch waste as substrate. International Journal of Chemical and Pharmaceutical Sciences. 5(2), 85-89.

2. Markou, G., Georgakakis, D., 2011. Cultivation of filamentous cyanobacteria (blue-green algae) in agro-industrial wastes and wastewaters: A review. Applied Energy. 88, 3389-3401

3. Federici F., Fava F., Kalogerakis N., Mantzavinosc D (2009) Valorisation of agro-industrial byproducts, effluents and waste: concept, opportunities and the case of olive mill wastewaters. J Chem Technol Biotechnol 84(6):895-900.

4. Lee, B.H., Kim, H.J., Yu, W.R., 2009. Fabrication of long and discontinuous natural fiber reinforced polypropylene biocomposites and their mechanical properties. Fibers and Polymers. 10(1), 83-90.

5. Yoon J.S., Sung A.J., Kyung B.S., (2011) Preparation and Mechanical Properties of Rice Bran Protein Composite Films Containing Gelatin or Red Algae. Food Sci. Biotechnol 20(3):703-707.

6. Raquez, J.M., Deleglise, M., Lacrampe, M.F., Krawczak, P., 2010. Thermosetting (bio) materials derived from renewable resources: A critical review. Prog Polym Sci. 35, 487-509

7. Jan, K., Riar, C.S., Saxena, D.C., 2015. Engineering and functional properties of biodegradable pellets developed from various agro-industrial wastes using extrusion technology. J Food Sci Technol 52(12), 7625-7639.

8. Simone, M., Leal, R., Evelise, F.S., Carlos, A.F., Sonia, M.B.N., 2009. Studies on the Properties of Rice-Husk-Filled-PP Composites - Effect of Maleated PP. Mat. Res 12(3), 333-338.
9. Wu, Q., Sakabe, H., Isobe, S., 2003. Processing and properties of low cost corn gluten meal/wood fiber composite. Ind. Eng. Chem. Res. 42(26), 6765-73.

10. AOAC. (1995) Official methods of analysis. U.S.A. Assoc of Official Anal Chem, Washington.

11. Anderson, R.A., Conway, H.F., Pfeifer, V.F., Griffin, E.L., 1969. Gelatinization of corn grits by roll and extrusion cooking. Cereal Sci. Today, 14, 4

12. Schettini, E., Santagata, G., Malinconico, M., Immirzi, B., Mugnozza, G.S., Vox, G., 2013. Recycled wastes of tomato and hemp fibres for biodegradable pots: Physico-chemical characterization and field performance. Resources, Conservation and Recycling. 70, 9- 19.

13. Rosentrater, K.A., Muthukumarappan, K., Kannadhason, S., 2009. Effects of ingredients and extrusion parameters on aquafeeds containing DDGS and potato starch. J. Aquacult. Feed Sci. Nutr. 1(1), 22-38.

14. Razzaq, M.R., Anjum, F.M., Khan, M.I., Khan, M.R., Nadeem, M., Javed, M. S., Sajid, M.W., 2012. Effect of temperature, screw speed and moisture variations on extrusion cooking behavior of Maize (Zea mays. L). Pak. J. Food Sci. 22(1), 12-22.

15. Cain, J., 2002. An alternative technique for determining ANSI/CEMA standard 550 flowability ratings for granular materials. Powder Handling and Processing, 14(3), 218-220.

16. Olajide, J.O., Igbeka, J.C., 2003. Some physical properties of groundnut kernels. J. Food Eng. 58, 201-204 BSI. (2010). EN 15210-15211:2009-solid biofuels-determination of mechanical durability of pellets and briquettes. British Standards Institution, London.

17. BSI., (2010). EN 15210-15211:2009- solid biofuelsdetermination of mechanical durability of pellets and briquettes. British Standards Institution, London.

18. Nalladurai, K, Morey, V.R., 2009. Factors affecting strength and durability of densified biomass products. Biomass Bioenerg. 33(3), 337-359

19. Gontard, N., Guilbert, S., Cuq, J.L., 1993. Water and glycerol as plasticizer affect mechanical and water vapor barrier properties of an edible wheat gluten film. J Food Sci 58(1), 206-211.

20. Gennadios, A., Weller, C.L., 1994. Moisture adsorption by grain protein films. Transactions of ASAE, 37(2), 535-539.

21. Shaw, N.B., Monahan, F.J., O’Riordan, E. D., O'Sullivan, M., 2002. Physical properties of WPI films plasticized with glycerol, xylitol, or sorbitol. J. Food Sci. 67(1), 164-167.

22. Parisa, F., Kurt, A.R., Kasiviswanathan, M., Mehmet, T., 2013. Effects of Steam, Moisture, and Screw Speed on Physical Properties of DDGS-Based Extrudates. Cereal Chem. 90(3), 186-197. 